\title{
¿Es adecuada, para la justicia global, la concepción política del "consenso sobrepuesto"?
}

\author{
Karl-Otto Apel
}

1. La retirada de la filosofía de la filosofía política contemporánea

El título de mi discurso podría ser elucidado, tentativamente, por otro que se lee como sigue:

“¿Podría una concepción pragmático-política de un acuerdo democrático, reemplazar cualquier fundamento filosófico universalista de justicia global e intercultural?"

Ambos títulos se relacionan, obviamente, con una tendencia a emancipar a la política de la filosofía; una tendencia que es una reacción al pluralismo de doctrinas metafísicas (o religiosas) comprensivas, con las que nos confrontamos hoy día a un nivel global, e incluso, en el ámbito de las democracias nacionales. De hecho, mi primer título se refiere a la filosofía del Liberalismo político, perteneciente a la época tardía de John Rawls, especialmente, en su ensayo de 1985, La justicia como justicia: politica y no metafísica.

El segundo título se refiere a la famosa (si no es que notoria) publicación, La prioridad de la democracia sobre la filosofía, publicada por R. Rorty como una interpretación de la posición asumida por Rawls en su época tardía.

¿Es adecuada, para la justicia global, la concepción politica del "consenso sobrepuesto"? 
El aspecto antifilosófico o antifundamental de la tendencia que quiero discutir se ha expresado más claramente y de modo más provocativo, así lo espero, con la pregunta de mi segundo título, y esto corresponde a la tesis planteada en el ensayo de Rorty que, en su primera publicación de 1988, hizo un ostentoso despliegue, particularmente, al proveer una interpretación del planteamiento de Rawls de una filosofía universalista sobre la justicia. Debo confesar que yo también me sentí incómodo con la pretensión hermenéutica de Rorty. Sin embargo, más tarde, al leer el ensayo de Rawls de 1985, tuve que admitir que Rawls, intencionalmente o no, a través de su nueva filosofía de Liberalismo político, se colocó en una posición aporética, cuyas implicaciones antiuniversalistas sólo han sido liberadas de todo escrúpulo por parte de Rorty. Por esta razón, comenzaré por lo que sigue a un argumento con Rorty quien, en nuestra época, representa la intención de un distanciamiento neopragmatista, no sólo de la metafísica, sino de todos los reclamos universales de validación de la filosofía. Intentaré mostrar que la defensa de la posición de Rorty no trata sólo de cuestionar la filosofía a través de la filosofía misma, sino que, en la situación actual de la globalización y de un posible choque de culturas, eso tendría, por obligación, unas consecuencias políticas desastrosas.

Luego de mi argumentación de Rorty, y a la luz de la misma, me acercaré a la posición de Rawls, e intentaré demostrar que su nueva filosofía de Liberalismo político es, sin duda, profundamente ambigua y ambivalente. Aunque se mantenga en su posición de "razonabilidad" esto no concurre ni con la interpretación hecha por Rorty, ni con su propia afirmación de dejar de lado la filosofía (moral). Sólo puede ser redimido $-\mathrm{y}$ esta constituye mi tesis final-, si pudiera resolverse el problema de un fundamento último de una ética universalista de la justifica, que en la Teoría de la justicia de Rawls, de 1971, no fue resuelta. Tendría que resolverse, a pesar de todos los prejuicios de la filosofía contemporánea - a través de un tipo de filosofía trascendental que no es idéntica a la metafísica comprensiva que depende de la cultura, en el sentido que la plantea Rawls.

\section{El salto de Rorty del liberalismo hacia el centralismo cultural, a través del relativismo histórico}

En su ensayo La prioridad de la democracia sobre la filosofia, Rorty parte de un supuesto, que es muy correcto, en mi opinión: De acuer- 
do con el liberalismo político, el Estado constitucional democrático tiene que asumir una posición de neutralidad ante todos los reclamos de la filosofía, así como con los reclamos de las confesiones religiosas y otras tradiciones de orientación valorativa. $\mathrm{Ni}$ es su obligación, ni le urge juzgar la verdad de las "visiones comprensivas", según formula Rawls la misma afirmación. Sin embargo, desde este supuesto correcto, Rorty deduce la conclusión excéntrica de que la base filosófica o la justificación de la posición política del Estado constitucional liberal no tiene sentido: ni hace falta, ni es posible. Desde la neutralidad filosófica del Estado liberal, se desprende, por cuenta de Rorty, que los filósofos que confiesan ser demócratas, deben suponer que la tradición cultural que pertenece a los Estados liberal-demócratas de Occidente, especialmente Estados Unidos, es el "contingente", pero, no obstante, rara vez posible, constituye la base de consentimiento para toda la discusión sobre cuestiones políticas.

Esto significa que los filósofos no deben tratar de defender la democracia y su neutralidad hacia las "visiones de mundo comprensivas", contra los posibles oponentes de su tradición, apelando a los argumentos filosóficos independientes y universalmente válidos, aunque podrían muy bien, según Rorty, tratar de propagar "de modo persuasivo" la tradición democrática occidental, incluyendo hasta su "utopía". Pero, en el caso de la confrontación con los oponentes que no comparten las presuposiciones de la tradición democrática - Rorty menciona a Nietzsche y a Loyola, aunque también hubiese podido pensar en Khomeini o hasta Chatami, o los presidentes de China, Singapur y Malasia-, "la persuasión" podría llegar a su límite. En tal caso, sugiere Rorty, los de Occidente deberían pensar como sigue:

...nosotros, herederos de la Iluminación, pensamos sobre los enemigos..., para usar la palabra de Rawols, "locos"... de la democracia liberal... No están locos porque bayan equivocado la naturaleza bistórica de los seres humanos. Están locos, porque los limites de la sanidad se definen por aquello que nosotros podemos tomar en serio. Esto, por otro lado, está determinado por nuestra crianza, por nuestra situación bistórica (pp. 187-8).

Un poco antes en su ensayo, Rorty afirma: "No debemos recurrir a los derechos humanos universalmente válidos (porque esto supondría disfrutar de los beneficios de la metafísica sin asumir las corres-

¿Es adecuada, para la justicia global, lo concepción política del "consenso sobrepuesto"? 
pondientes responsabilidades)", sino que "...necesitaríamos algo que haga la distinción entre una cierta conciencia individual que respetamos, y la que condenamos como "fanática". Esto sólo puede ser algo relativamente local y etnocéntrico: la tradición de una comunidad en particular, el consenso de una cultura particular. De acuerdo con esta visión, lo que es válido como racional o como fanático, es algo relativo al grupo ante el cual pensamos que es necesario justificarnos - es decir, al cuerpo de las creencias compartidas que determinan la referencia de la palabra "nosotros"." (pp. 176s)

Es mi opinión que es difícil encontrar argumentos en la filosofía occidental contemporánea que desafíen la argumentación de Rorty y que se les haga fácil a los fundamentalistas y nacionalistas de todo tipo a que defiendan su centrismo cultural o etnocentrismo contra el ideal occidental de la democracia liberal y los derechos humanos. Lo que muestra Rorty, de hecho, es que el relativismo histórico, que se reduce a un centrismo cultural a través de la proclamación de la propia tradición como la base única de consentimiento para un diálogo intercultural, rápidamente reduce, de modo absurdo, aquel liberalismo al cual está alegadamente conectado. En el contexto de una práctica política esto desembocaría, sin duda, en una posición que es, por lo menos, incompatible con el establecimiento de una sociedad multicultural - sea al nivel cosmopolita o al nivel regional de la coexistencia humana bajo una ley de justicia comúnmente reconocida-.

No continuaré aquí discutiendo la posición de Rorty, pero regresaré al hecho de que Rorty afirma, en el ensayo que cité - La prioridad de la democracia sobre la filosofía-, que interpreta la posición del Rawls de la etapa tardía. Es una actitud totalmente historicista y antiuniversalista la que Rorty le coloca también a Rawls. Para sus propósitos de una "teoría política sobre la justicia", Rorty sugiere que Rawls "no necesita del "socratismo", es decir, de la idea de que cualquiera que esté dispuesto a escuchar la razón — de escuchar todos los argumentos- pueda ser traído de vuelta a la verdad, porque el ser humano tiene un centro (una chispa divina, o una capacidad de trayectoria de la verdad, llamada "razón"), y... la argumentación, dado el tiempo y la paciencia, penetrará su centro... Somos libres de ver el descentralismo del ser como un contingente histórico a través de todo el camino" (p. 188). Por tanto, en cuanto se refiere a Rorty, Rawls "puede estar contento con la noción del ser humano como una red 
descentrada de creencias y deseos históricamente condicionados y, por tanto, podría asumir igualmente que un acuerdo democrático sobre la justicia "deberá llevar a cualquier equilibrio intersubjetivo reflexivo capaz de ser logrado, dado el montaje contingente de los sujetos en cuestión" (p. 191).

Contraria a mi primera reacción al leer el ensayo de Rorty, hoy me inclino más - lo dije ya- a no considerar la pretensión de Rorty de interpretar a Rawls como algo totalmente extravagante. Por tanto, intentaré, en lo que sigue, de reconstruir, a la luz de la perspectiva de Rorty, el desarrollo de la propia interpretación de Rawls sobre la "Teoría de la justicia", aún tomándome el riesgo de ofrecer un recuento muy selectivo y simplificado.

\section{El "liberalismo político" de Rawls: Un intento paradójico de con- servar el universalismo de una teoría de la justicia, al evitar un fundamento filosófico}

En la versión original (1971) de la Teoría de la justicia de Rawls, se podría encontrar el problema no resuelto del fundamento. No se proveyó el fundamento adecuado para esta teoría, según apuntaba el mismo Rawls en varias ocasiones posteriores, a través de una "opción racional" (en el sentido de la teoría de decisiones y juegos) de los partidos que concluyen el contrato en la "posición original". (Esto hubiera implicado que la teoría de la justicia, en su totalidad, habría fracasado desde el principio, al estar sujeta a la aporía de Hobbes, en cuanto que "el sentido de la justicia" - es decir, de mantener el contrato y no concluirlo a expensas de los partidos excluidos- no puede derivarse del autointerés estratégico calculado de aquellos que concluyen un contrato). El fundamento de la teoría de Rawls estaba contenida, más bien, en aquellas presuposiciones con las que el mismo Rawls - -en su llamado "constructivismo kantiano" de su época tardía- se había comprometido ingeniosamente, desde el principio, ya que impuso ciertos límites a la posición original (tal como "el velo de la ignorancia" relacionado con la posición social de los votantes en orden a que se optara por la justicia).

Estos límites se imponían para obligar a los votantes originales a que se imaginaran en la posible situación de cada posible ciudadano y, por tanto, como votantes libres y equitativos, a que optaran por aque-

¿Es adecuada, para la justicia global, la concepción políica del "consenso sobrepuesto"? 
Ilo que pudiera ser estratégicamente "racional" y, a la vez, "razonable" en el sentido de la ejemplificación de la "justicia como justicia".

Sin embargo, equé sucede, entonces, con el fundamento de la idea de Rawls sobre "razonabilidad", es decir, de la justicia como justicia, comprometida por los límites de la "posición original"? El fundamento último de su teoría, lo podía ofrecer Rawls, sólo a través del recurso del "equilibrio reflexivo", que podía ser alcanzado por un procedimiento de corrección mutua entre "el sentido de justicia" del filósofo y "el sentido común de la justicia" (que incluiría hasta el de los votantes "en la posición original"). Con esta sugerencia, Rawls apeló, me parece a mí, a un artefacto que es inevitable, de hecho, en cada caso de intento de explicación de significados, relacionados con conceptos que todavía no se han definido. A estos efectos, hay una cercana afinidad entre el concepto del "equilibrio reflexivo" de Rawls y el "círculo del entendimiento" de la "filosofía hermenéutica" (en el sentido de Dilthey, Heidegger y Gadamer). Pero en nuestro concepto de búsqueda de la justicia global, con el contrapeso universalista -viz. justicia intercultural-y la búsqueda de la interpretación hermenéutica de la propia tradición particular, resulta muy débil el apelar al "sentido común" para lograr un fundamento último de justicia. Esto no es capaz de movilizar la resistencia de una medida independiente contra el impacto de la propia tradición cultural, que goza, de todas maneras, de una prioridad genética en el preconocimiento propio de la vida del mundo. Este hecho -es decir, la tendencia a caer como víctimas de un bistoricismo (relativismo) - ha sido evidenciado por el desarrollo de la autointerpretación de Rawls de un modo similar al de las filosofías de Dilthey, Heidegger y Gadamer. Permítanme que muestre esto.

En su ensayo de 1985 - La Justicia como justicia: política y no metafísica-, Rawls asume la siguiente autointerpretación:

El punto general es éste: como asunto práctico, ninguna concepción moral general puede proveer la base para una concepción pública de la justicia en una sociedad democrática modema. Las condiciones sociales e bistóricas de tal sociedad tienen sus origenes en el desarrollo del principio de tolerancia y en el crecimiento de un gobierno constitucional y la institución de las grandes economias de mercado. Estas condiciones afectan profundamente los requisitos de una concepción práctica de la justicia politica: tal concepción práctica debe permitir la diversidad de doctrinas y una pluralidad de concepciones conflictivias 
$e$ inconmensurables del bien afirmada por los (as) miembros de las sociedades democráticas existentes. (P. 225, énfasis añadido)

En este aspecto, Rawls disputa el asunto de manera obviamente similar a Rorty —una teoría política puede tener su base (es decir, el fundamento de su validez) en una concepción "filosófica" de "moralidad”. En su lugar, él recurre a la génesis bistórica de las condiciones de las sociedades democráticas de Occidente. Pero, equé sucede con la afirmación previa de Rawls que se refiere a una concepción "que se sostiene sola" sobre el "derecho", que tiene que ser prioritaria sobre la "pluralidad" de concepciones inconmensurables del bien, en una sociedad democrática moderna? (Siempre he considerado esta afirmación de la "Teoría de la Justicia" de Rawls, como una confirmación de la concepción de Habermas y la mía propia, de una complementariedad entre una filosofía universalista de la justicia y las muchas concepciones particulares de la buena vida a las que pueden optar los individuos y comunidades dentro de una sociedad liberal y multicultural.) Precisamente, la respuesta a esta pregunta la da, sugiero, la concepción tardía de Rawls sobre "consenso sobrepuesto", que dice:

La justicia como justicia es una concepción política, en parte porque comienza desde una tradición politica particular. Esperamos que se sustente, al menos, por lo que me gustaría llamar "consenso superpuesto", que es un consenso que incluye todas las doctrinas filosóficas $y$ religiosas controvertibles que, presumiblemente, persistirán y encontrarán adberentes en una sociedad constitucional democrática más o menos justa.

La concepción de "consenso sobrepuesto" en la Teoria de liberalismo político parece constituir un equivalente de la concepción de "equilibrio reflexivo" de su más antigua Teoría de la Justicia. Esto aparece más claro a partir de la discusión de Rawls con J. Habermas en 1995. El aspecto principal de esta discusión, sugiero, se ilustra con el hecho de que Rawls, considera que hasta la concepción discurso-teórica y procedimentalista de la justicia de Habermas, es una "doctrina comprensiva", y que, por tal razón, no podría tener la esperanza de ser un "consenso sobrepuesto" en una sociedad pluralista. Su propia concepción, y aquí concede Rawls, "no llega a ser... propiamente formal y verdaderamente universal y, por tanto, ser parte de las presuposiciones cuasi-trascendentales (como a veces dice Habermas) establecidas por la teoría de acción comunicativa".

281

¿Es adecuada, para la justicia global, la concepción políica del "consenso sobrepuesto"? 
Ya en este aspecto podría haber objeciones: Aún cuando la concepción de Habermas es una doctrina comprnsiva dependiente de la cultura, ¿qué pasa, entonces, con la concepción de Rawls que, expresamente, recurre a la tradición occidental de instituciones? O, ¿cuál sería el criterio legitimizador para la concepción de Rawls sobre "consenso sobrepuesto"? En su discusión con Habermas del año 1995, Rawls, de modo muy rígido, apunta a su intención de emancipar la teoría política de los fundamentos filosóficos:

La idea central es que el liberalismo político se mueve dentro de la categoría de lo político y deja lo filosófico tal cual. Deja sin atender toda clase de doctrinas, religiones, metafísicas y morales, con sus largas tradiciones de desarrollo e interpretaciones. La filosofía política continúa separada de tales doctrinas y se presenta bajo sus propios términos como autónoma. Por tanto, no puede argumentar su caso a través de la invocación de ninguna doctrina comprensiva, ni a través de su crítica, ni a través de su rechazo...

Más tarde, Rawls ofrece una reformulación y otra explicación de su idea central, que resulta especialmente interesante, ya que parece sugerir una respuesta sofisticada a la pregunta de cómo puede relacionarse la concepción política autónoma con las "doctrinas comprensivas":

Puede formularse independientemente de cualquier doctrina comprensiva particular, religiosa, filosófica, o moral. Mientras que suponemos que puede derivarse de, o ser apoyada por, o de alguna otra manera relacionarse a una o más doctrinas comprensivas (de becho, esperamos que si pueda relacionarse con muchas de tales doctrinas), no se presenta como dependiente de, o como presuponiendo, ninguna de tales visiones.

No obstante, aún luego de esta explicación, se hace todavía difícil decir qué constituye una autoridad "autónoma" de legitimización de la justicia por consenso sobrepuesto.

De cara a todas las características de la reformulación de la teoría de la justicia en el Liberalismo político de Rawls, resulta muy sugerente concebir la concepción del "consenso superpuesto", simplemente como el artefacto de un pacto pragmático-político. Por dejar de lado todo fundamento moral filosófico de la justicia, parece ser que se rinde ante las circunstancias contingentes de una constelación de visiones comprensivas en la situación particular de una sociedad pluralista. Yo 
sugiero que hay muchas razones de fuerza para tal interpretación de la nueva concepción de Rawls.

Por ejemplo, el hecho de que él ahora -junto con los "comunitaristas" - comparte la opinión de que todos los posibles fundamentos morales pueden ser trazados hacia atrás, hasta doctrinas culturalmente dependientes, metafísicas o religiosas; más aún, el hecho innegable de que, en nuestros días, la política debe poder manejar de alguna manera el problema de asegurar un orden de ley común en y para una sociedad pluralista, o hasta para una sociedad multicultural.

Finalmente, quisiera mencionar que cualquier teoría de consenso (de una posible redención de los reclamos normativos de validez), si sólo apunta al alcance de un consenso fáctico (un acuerdo) en espacio y tiempo, sin estar orientado, a la vez, hacia la posibilidad de cuestionar cualquier consenso fáctico a la luz del "principio regulador" (Kant) de un consenso ideal ( $\mathrm{y}$, hasta ahora, arraigado moral y filosóficamente), debe poder entregarse a las circunstancias contingentes de una situación histórica, tal como lo hace cualquier pacto pragmático-político.

A través de este último comentario, creo haber marcado la deficiencia filosófica que va a la par con la diferencia entre un "consenso sobrepuesto", concebido como un pacto pragmático-político y un principio genuino de universalización de normas morales. Pero, para poder demostrar que esta deficiencia filosófica también tiene consecuencias para una praxis social y política, trataré de elucidarlo a través de la utilización de un ejemplo: En el mundo antiguo, durante la época de Platón o, más tarde, durante la época del apóstol Pablo, es decir, todavía dentro del Imperio Romano, que estaba basado sobre una sociedad multicultural que incluía muchas religiones diferentes y tradiciones de valores metalísicos, no habia la posibilidad de lograr un "consenso sobrepuesto", relacionado con la injusticia de la esclavitud. (Todavía más tarde, en el siglo Xvı, el teólogo escolístico, Sepúlveda, en su controversia con Bartolomé de las Casas sobre el trato justo de los indios de las colonias españolas, podía apelar al "consenso sobrepuesto" antiguo y a los argumentos de Aristóteles a favor de la esclavitud).

¿Podría excluirse, de nuestro mundo actual, tal discrepancia entre los resultados de un "consenso sobrepuesto" fáctico y el principio regulador y universalizante de la filosofía moral?

¿Es adecuada, para la justicia global, la concepción politica del "consenso sobrepuesto"? 
Yo no lo creo. En relación con los problemas de la justicia global hoy, yo pensaría, en primer lugar, sobre la exclusión de estas discusiones de los millones de pobres del Tercer Mundo, a través de los cuales pudiera establecerse, al nivel de la política, un "consenso sobrepuesto" sobre un orden justo de ley y economía (Este es un aspecto que trajo a la luz en las últimas décadas el filósofo de la liberación, Enrique Dussel).

En otro sentido, que no deja de tener conexión con el primero, yo pensaría sobre el hecho de que, también, los intereses de las generaciones futuras son todavía, más o menos, excluidos de nuestros procesos políticos sobre formación de consenso (Parece ser que, hasta los temas ecológicos de estos procesos han perdido relevancia en los últimos años). Pero antes de que siga con este aspecto de posible crítica, debo mencionar que el mismo Rawls no parece aceptar el que se equipare su concepción sobre "consenso sobrepuesto" con el pacto programático-político que yo he intentado considerar hasta ahora.

Por tanto, en esta discusión con Habermas, Rawls sugiere una distinción entre "dos ideas diferentes de consenso", que él caracteriza del siguiente modo: "Una idea de consenso viene de la política de cada día, donde el gusto del político viene de alcanzar acuerdos... Esta idea de consenso es la idea de una superposición que ya está presente o latente y que podría articularse a través de la destreza del político de conjuntar los intereses existentes y que el político conoce intimamente".

Este tipo de consenso, que se acerca a nuestra interpretación sugerida, es "política del modo equivocado", según Rawls, porque no satisface el criterio de ser "razonable". Por tanto, Rawls sugiere otra concepción:

La idea muy diferente del consenso en el liberalismo político -..-La idea que yo llamo un consenso sobrepuesto razonable- es que lat concepción política de la justicia se maneja como una visión autónoma que puede ser justificada pro tanto, sin mirar cómo, o sin intentar acoplar, o ni siquiera conocer cuáles son las doctrinas comprensivas existentes (Th. 39f). Trata de no poner obstáculos en el camino de todas las doctrinas razonables que apoyan una concepción politica, a través de la eliminación, en dicha concepción, de cualquier idea que vaya más allá de lo político, y que no toda doctrina razonable pudie- 
ra esperarse que apoye razonablemente. (Hacer esto violaria la idea de mutualidad). Cuando una concepción política cumple con estas condiciones y es, además, completa, asi lo esperamos, las doctrinas comprensivas razonables, afirmadas por ciudadanos responsables, pueden apoyarla, y tendrán la capacidad de conformar estas doctrinas bacia si mismas. (Th. IV:6-7)

Esta aclaración es, de hecho, muy informativa, pero, en el contexto de nuestro problema, parece que se mueve por la fuerza del peso de la justificación, desde la nueva concepción de "consenso sobrepuesto", hacia la justificación de la "concepción de justicia autónoma" o "el derecho", que conocemos de la Teoría de la Justicia. Porque si hay que resolver y "justificar pro tanto primero" el asunto de la concepción de la justicia, sin buscar las teorías comprensivas existentes, no podemos - parece- asumir ningún criterio de justicia política a partir de las teorías comprensivas existentes, sino que debemos asumir el recurso del criterio formal de un ideal y, por tanto, del consenso universal concebido por la filosofía moral.

Esto parece confirmarlo el mismo Rawls, ya que él sugiere que la complementación de la teoría de la justicia con el "consenso sobrepuesto" dentro del marco del "liberalismo político", es sólo una segunda etapa de la totalidad de su teoría política de justicia, una etapa que presupone ya la elaboración de la concepción "autónoma" de la justicia en una primera etapa, pero que se hace necesaria ella misma como respuesta a la pregunta de cómo pucde asegurarse la "estabilidad" de la sociedad democrática bien ordenada.

Sobre este problema se pronuncia Rawls:

...el tipo de estabilidad que se requiere de la justicia como justicia, se fundamenta, pues, en que es una visión política liberal, que quiere ser aceptable para los ciudadanos, como razonable y racional; a la vez que libre y equitativa $y$, por tanto, dirigida a su razón pública...

Hasta el momento, entiendo yo, se articula nuevamente la presuposición de las categorías principales de la "teoria de justicia" del año 1971, pero se le añade la complementación de la nueva concepción:

La justicia como justicia no es razonable en un primer momento, a menos que, de manera adecuada, pueda ganarse el apoyo de la razón de cada ciudadano, explicada dentro de su propio marco.

285

¿Es adecuada, para la justicia global, la concepción politica del "consenso sobrepuesto"? 
Supongo que esta concepción deberá ser capaz de imponer restricciones, tanto sobre los consensos sobrepuestos de las doctrinas comprensivas existentes a través de la presuposición de la "concepción autónoma de justicia como justicia”, como sobre esta concepción de la primera etapa, tal como está elaborada, hasta el punto de ser aceptada por las doctrinas comprensivas existentes. Hasta ahora, me parece que todo se reduce a la necesidad de una adecuada teoría filosófica de consenso, que se fundamente, primariamente, no en razones pragmático-políticas, sino sobre un reclamo de la razón pública que se lleve a cabo bajo las condiciones de reconocimiento mutuo de todos los ciudadanos, de ser igualmente libres y razonables y, más aún, bajo la condición de estar abierta a posibles correcciones, por objeción, por parte de los representantes no-presentes de las visiones comprensivas. Esto no significaría, sugiero, que la necesidad política de alcanzar un consenso fáctico una y otra vez, con miras a asegurar la "estabilidad" de un orden democrático y su reproducción, podría, o debería, ser eliminada. Pero, sí significaría, ciertamente, que las condiciones normativas para alcanzar un consenso razonable, y, por tanto, también la posibilidad de cuestionar cada consenso fáctico deberá estar arraigada filosóficamente, digamos, por una concepción de consenso ideal que pudiera servir como un principio regulador procedimental para buscar y también para cuestionar un "consenso sobrepuesto".

Pero esta sugerencia de regresar a una concepción filosófica que - sugiero- estaría de acuerdo con la "Teoría de Justicia" original, Rawls la niega expresamente. Por lo mismo, en su discusión con Habermas, él niega para sí mismo el despegue de una "concepción moral general", que pudiera proveer el fundamento para una "concepción pública de la justicia en una sociedad democrática moderna". En su lugar, él quiere tomar como punto de partida algunas ideas centrales de justicia política, intuidas o sugeridas en la tradición de la democracia de Occidente y unir estas ideas en una concepción coherente. En este sentido, coloca su visión en contraposición con la visión de Habermas, diciendo que es "sustantiva y no procedimental". Sin embargo, para evitar una concepción que es "política en un sentido falso", Rawls ahora clama por una concepción de "extender" sus ideas sustantivas a partir de la tradición de Occidente. Él explica su visión como sigue:

...aun cuando tal concepción no se sostiene para todas las sociedades en todo momento y lugar, no es, por tanto, todavia, histórica o 
relativista; sino que es universalista, en cuanto es posible extenderla, de forma apropiada, hacia una concepción de justicia razonable para las relaciones entre todas las naciones.

En estas últimas aseveraciones, Rawls, obviamente, se refiere a su contribución ante los Discursos de Amnistía de Oxford sobre Derechos Humanos de 1999, bajo el título "La ley de los pueblos". Aquí, Rawls, ciertamente intentó, por primera vez, sacar las consecuencias de su concepción de justicia política para manejar los problemas internacionales que surgen por el hecho temático de la globalización. Intentemos, entonces, capturar y acceder al método de Rawls de "extender" universalmente su concepción primordialmente occidental, de "consenso sobrepuesto".

4. "La ley de los pueblos" de Rawls: Un intento de manejar la globalización de la justicia política a través de la extensión de una concepción occidental de la misma

Primeramente, quisiera enfatizar que aprecio mucho la declaración preliminar en el ensayo de Rawls, ya que me parece que expresa un gran avance introspectivo, más allá de la ingenuidad usual de la ecuación occidental sobre los problemas de justicia, con aquellos de una teoría doméstica de la democracia que se abstrae de todo el problema de política extranjera. Rawls expresa justamente:

Cada sociedad debe tener una concepción de cómo se relaciona con otras sociedades y de cómo debe comportarse hacia ellas... Debe poder formular ciertos ideales y principios para guiar sus políticas hacia otros pueblos.

Ahora bien, Rawls también apunta, de modo muy claro, que, de acuerdo con su método de extensión, su punto de partida es distinto al de la filosofía formalista. Sobre esto, afirma:

Una visión constructivista... no comienza en todos los casos con la legitimación de la autoridad de los principios universales primeros. Una doctrina liberal constructivista es universal en su capacidad de alcance, una vez la misma se extienda para ofrecer principios a todos los sujetos políticos relevantes, incluyendo una ley de los pueblos para el sujeto más comprensivo: la sociedad politica de los pueblos. Su autoridad reside sobre los principios y concepciones de la razón prác-

¿Es adecuada, para la justicia global, la concepción politica del "consenso sobrepuesto"? 
tica, siempre y cuando sean ajustados adecuadamente para que puedan ser adaptados a diferentes sujetos según van emergiendo en secuencia...

Esta última distinción de las teorías comprensivas tradicionales, se explica más claramente en el siguiente párrafo:

el constructivismo asume... que bay otras formas de unidad, aparte de aguellas definidas completamente por los principios generales primeros que forman un esquema coberente. La unidad puede darse también a través de una secuencia con la comprensión de los principios dirigidos al sujeto de cada acuerdo subsiguiente, han de estar subordinados a aquellos [principios] dirigidos a sujetos de todos los acuerdos previos; o si no, coordinados con y ajustados a ellos por ciertas reglas de prioridad.

Pero, no obstante este anuncio de perseguir un método para manejar a los distintos sujetos de acuerdo a una secuencia de casos, Rawls excluye, desde el principio, a ciertos sujetos como no pertenecientes a los posibles casos de una teoría "normal" o "ideal" de una ley de los pueblos. Tal situación, como dato interesante, Rawls la da por sentado cuando tenemos que manejar el problema de "cómo las sociedades más pobres y menos avanzadas tecnológicamente del mundo, podrían acceder a las condiciones históricas y sociales que les permitan establecer instituciones justas que funcionen..." 36 (sic).

Pero, más aún, para Rawls también, en aquellos casos de "sociedades forajidas" o "tiránicas" y "dictaduras", éstas pertenecen a los sujetos de una "teoría no-ideal" que tiene que poder manejar aquellos casos de "condiciones no favorables".

Esta exclusión primaria de sujetos de una teoría "no ideal", que, yo creo, resulta sorprendente para una concepción no formalista, probablemente se introduce por Rawls, porque, como ya sabemos, él quiere evitar la equivalencia de la concepción de "consenso sobrepuesto" con la de un instrumento pragmático-político que depende de circunstancias contingentes. Tal instrumento es, por supuesto, necesario -creo, hasta como un requisito de la ética política de la responsabilidad- para todas "las condiciones no favorables".

Por su teoría “ideal" de la "ley de los pueblos", Rawls está preparado para proveer la posibilidad de una coexistencia entre las sociedades 
"liberal-demócratas" y "no-liberal-demócratas", es decir, de aquellas sociedades "jerarquizadas”. Esta concesión, por así decirlo, edifica el aspecto de la parte ideal de su proyecto de "extensión". ¿Cómo puede, pues, aplicar Rawls, su método de extensión a través de la construcción de una secuencia de casos, a la relación entre los "liberal-demócratas" y las sociedades "jerárquicas”, pero bien ordenadas?

El argumento principal de Rawls para la admisión necesaria de sociedades "no-liberales" pero "bien ordenadas" es la "subordinación" de este caso de "consenso sobrepuesto" al primer caso, que se ejemplifica a través de la "teoría de justicia" original; esta subordinación puede hacerse por un cierto argumento de analogía.

La nueva teoría puede también ser liberal, según Rawls, al aplicar e] principio de liberalismo que subyace al "consenso sobrepuesto" dentro de una sociedad democrática, a "la sociedad de sociedades políticas": "Como antes, los partidos [en la posición original] son representativos, pero ahora son representativos de los pueblos cuyas instituciones básicas satisfacen los principios de justicia seleccionados en el primer nivel"; por tanto, resulta, según Rawls, "que las sociedades liberales y las jerárquicas pueden estar de acuerdo en la misma ley de los pueblos".

Ahora bien, la admisión de "sociedades jerárquicas" incluiría una reducción considerable de los "derechos humanos" —según Rawls, en particular, a una cancelación del derecho "de libre expresión" [Yo comentaría que esto implicaría todos aquellos derechos que se refieren a la igualdad de participación en la esfera pública de la política, que Rawls, expresamente, reclamó para su concepción, en contra de las dudas planteadas por Habermas]. Mi pregunta es entonces: ¿Puede esta reducción de los "derechos humanos" ser justificada de modo plausible por el argumento de analogia [es decir, por la transferencia de la actitud liberal del nivel de los ciudadanos al nivel de los Estados]?

Mi respuesta es que, en principio, este procedimiento no puede justificarse. Esto implicaría que "la soberanía interna de un gobierno", al obligar a sus ciudadanos a una forma de totalitarismo religioso o secular, no podría criticarse de ninguna manera a través de la apelación a los derechos humanos. Seguramente, cste no es el punto de argumentación en la teoría de Rawls. Más bien, él sigue la intuición correcta al

¿Es adecuada, para la justicia global, la concepción políica del "consenso sobrepuesto"? 
formular ciertas condiciones incisivas encaminadas a tolerar los regímenes "jerárquicos". Por lo que puedo ver, el criterio decisivo en este contexto es el reclamo de que el "sistema de ley" en cuestión, "ipueda cumplir con los elementos esenciales de legitimación ante los ojos de su propia gente!"

Este es, ciertamente, un criterio plausible, pero, ¿por qué es plausible? Es obvio que el criterio no se colige de la estrategia de extensión, que está fundamentada sobre el argumento de analogía o de la transferencia liberal del nivel de las personas al nivel de las sociedades; más bien, nos lleva nuevamente al nivel de personas. Pero el nuevo criterio es también incompartible con el "consenso sobrepuesto", que se ha de alcanzar en el contexto de una "democracia liberal" de tradición occidental, hacia la cual se orientaba la concepción original de la Teoría de Justicia de Rawls. Esto hubiera hecho imposible aquel tipo de "extensión liberal" a la teoría global de "ley de los pueblos" que Rawls tenía en mente. Esta concepción última no puede subordinarse a los principios de casos tempranos de la secuencia de la extensión de teoría política de la justicia de Rawls.

\section{El fundamento trascendental de la justicia global por la ética del discurso}

La solución de este dilema, en mi opinión, sólo puede ser provista por un discurso reflexivo filosófico que subyace en toda concretización política -y, por tanto, bistórica y fáctica- del problema de la justicia como justicia: recurrir a las presuposiciones morales de aquel discurso primordial, a través del cual la filosofía tiene que discutir estos problemas. Encontramos aquí un punto de partida unitario para "una teoría general de justicia" y de "derechos humanos": un punto unitario básico que no es ni empirico, ni metafísico, sino trascendental. Esto, porque el principio que al final podría sustentar el criterio intuitivo de "legitimización" de Rawls para las sociedades liberal-demócratas y noliberal-demócratas, podría estar asentado sobre un postulado que siempre se reconoce a priori de derechos equitativos y corresponsabilidad equitativa en la solución de problemas de todos los involucrados en el discurso; esto contiene el reclamo de que todas las soluciones propuestas sobre una teoría de la justicia, deben llenar, idealmente, la condición de que puedan ser aceptables para todas las personas afectadas, y esto significa primariamente: para los ciudadanos de los Estados 
constitucionales y, antes y más allá, hasta para las personas que se encuentran fuera de los límites de los Estados constitucionales bien ordenados, como por ejemplo, los habitantes del llamado Tercer Mundo. No debe haber ningún acuerdo fáctico, a expensas de personas no representadas, pero sí afectadas.

Este reclamo fundamental es, por supuesto, sólo un "principio regulador" (en el sentido kantiano); puede ganar su contienda sólo por su correcta aplicación; y está arraigado en una reflexión que no es todavía política, sino filosófica; en el sentido de una ética del discurso. Se convierte en político a través de dos formas: de acuerdo al método ideal de procedimientos de aplicación, que es ya siempre reconocido en un discurso filosófico serio, la forma de establecer un orden político justo, es decir, un Estado constitucional, tiene que seguir, él mismo, el ideal del procedimiento del discurso (que hasta permitiría la negociación sólo bajo las precondiciones justas que puedan ser justificadas, a su vez, por el discurso). Por supuesto, esta forma no permitiría la formación de un consenso que fuera "político en un sentido falso", para citar a Rawls, sino que permitiria, en cualquier momento, criticar un "consenso sobrepuesto" fáctico y, por tanto, dependiente del contexto.

Pero también habría que considerar, desde el principio, una segunda manera de aplicación política sobre el fundamento de la ética del discurso. Ya que el principio de la ética del discurso implica el telos de perseguir un orden justo que pudiera ser aceptable para todas las personas afectadas, la responsabilidad política debe también cuidar de todas aquellas situaciones que no permitan soluciones de comunicación ideales relacionadas al problema de establecer un orden justo, hasta en aquellas situaciones donde los derechos bumanos sólo pueden ser defendidos a través de la guerra.

Este segundo modo para que la ética del discurso sea política, puede estar relacionado con la parte "no-ideal" de la concepción de Rawls, que tiene que poder manejar las "condiciones no favorables", según él afirma.

Ahora Rawls podría, presumiblemente, sospechar que mi recurso a la situación primordial del discurso filosófico deberá descansar sobre la metafísica, es decir, sobre alguna "doctrina comprensiva". Y, de hecho, soy un colega peor que Habermas, por el hecho de que recurro 
a un fundamento trascendental pragmático de la ética del discurso, sin ningún recurso cuasi empírico a la sociología o la lingüistica. Pero creo que sería un error fatal de nuestros tiempos el confundir el método radical de la reflexión trascendental sobre la validez de las condiciones de la argumentación con la metafísica. Como contraste, considero que es la única forma confiable de evitar cualquier metafísica, es decir, cualquier doctrina "comprensiva", dependiente de la cultura. 